\title{
Leaf transcriptome of oil palm (Elaeis guineensis Jacq.) infected by Ganoderma boninense
}

\begin{abstract}
Oil palm is susceptible to Ganoderma infection which causes basal stem rot (BSR). Induced defense gene profiles in oil palm leaves will assist the identification of markers for detection of infected oil palms. In this study, we have sequenced the mRNA samples from the leaves of G. boninense infected oil palm seedlings (LG) and in control treatment (LC). Differential gene expression analysis showed 711 and 482 genes that were up-and down-regulated more than fourfold in LG, respectively, compared to the LC. Differential gene expression analyses revealed the modulation of oil palm genes involved in defense response such as chitinases, glucanases, and thaumatin-like proteins that showed up-regulation in LG. In addition, genes for enzymes related to the biosynthesis of flavonoids, alkaloids, and terpenes were upregulated, while many genes involved in photosynthesis were found to be suppressed in LG. Our findings provided information on how infected oil palm leaves diverting their resources into defense at the cost of other biological processes, contributing towards identification of candidate markers for the detection of infected oil palms.
\end{abstract}

Keyword: Basal stem rot; Ganoderma; Leaf; Oil palm; Global gene expression 